\title{
Anthropomorphized species as tools for conservation: utility beyond prosocial, intelligent and suffering species
}

\author{
M. Root-Bernstein $\cdot$ L. Douglas $\cdot$ A. Smith $\cdot$ D. Veríssimo
}

Received: 5 October 2012/Accepted: 14 May 2013/Published online: 25 May 2013

(C) Springer Science+Business Media Dordrecht 2013

\begin{abstract}
Anthropomorphism has recently emerged in the literature as a useful tool for conservation. Within the current conservation literature, description of the development of anthropomorphisms and the range of species that can be anthropomorphized overlooks established and emerging evidence from anthropological and other social science studies of human-animal relationships. This research shows that people anthropomorphize a very broad range of species, including plants. We discuss how people construct anthropomorphic meanings around species, through a diversity of mechanisms and with both positive and negative effects. We then review the many gradations and forms of anthropomorphism, and some related conceptions in non-Western cultures, which have different types of utility for conservation. Finally we discuss cases where animals are anthropomorphized but with negative outcomes for human-animal interactions and conservation. Limiting the use of anthropomorphism in conservation to prosocial, intelligent, suffering animals risks suggesting that other species are not worthy of conservation because they are not like humans in the "right" ways. It would also mean overlooking the application of a powerful tool to the promotion of low-profile species with high biological conservation value. We emphasize that negative outcomes and conflicts with ecosystem-level conservation actions are also possible and need to be carefully managed. Use of anthropomorphism in
\end{abstract}

\footnotetext{
M. Root-Bernstein $(\bowtie)$

Department of Ecology, Pontificia Universidad Católica de Chile, Santiago, Chile e-mail: meredith.root-bernstein@ouce.ox.ac.uk

M. Root-Bernstein

Oxford University Centre for the Environment, Oxford University, Oxford, UK

L. Douglas

Department of Ecology, Evolution and Environmental Biology, Columbia University, New York, NY, USA
}

A. Smith

Department of Management, Monash University, Melbourne, VIC, Australia

D. Veríssimo

Durrell Institute of Conservation and Ecology, University of Kent, Kent, UK 
conservation must take into account how people engage with species and attribute value to their characteristics.

Keywords Anthropomorphism · Conservation · Egomorphism · Flagship ·

Non-human charisma

\section{Introduction}

Anthropomorphism is common in traditional and popular cultures, and is regarded as an important way in which people make sense of interactions with the non-human world (Guthrie 1997; Mitchell 1997; Lorimer 2007; Taylor 2011). Recently, the role of anthropomorphism as a useful tool for conservation outreach and environmental education has been gaining attention (Chan 2012; Tam et al. 2013). However, we believe that most conservationists still underestimate the breadth of applicability of anthropomorphism to conservation, and are likely to be unaware of research from the social sciences making clear anthropomorphism's potential as a powerful but double-edged sword.

One way in which anthropomorphism has been positioned as a scientifically respectable tool is through the recommendation that it be used only for animals that are similar to humans in ways validated by biological science. According to Chan (2012), to date the strongest argument can be made for the use of the following traits as the basis for empathetic anthropomorphism: being (1) prosocial, (2) intelligent, and (3) able to suffer. We agree that the perception of shared features can lead to the development of empathy (Mitchell 1997; Milton 2005; Lorimer 2007). However, social science research shows that engagements with a much broader set of features can form the bases of empathetic anthropomorphism and the impetus for conservation actions. We are also concerned that limiting the use of anthropomorphism in conservation to prosocial, intelligent, suffering animals risks suggesting that most species are not worthy of conservation because they are not like humans in the "right" ways. This would produce an anthropocentric, two-tiered conservation agenda favoring a very small percentage of biodiversity (excluding, for example, all plants). It would also mean overlooking the application of a powerful tool to the promotion of low-profile species with high biological conservation value, such as invertebrates. We argue that anthropomorphism should not be seen as a criterion that prioritizes species that more closely resemble humans in predefined ways, but as a strategic tool within conservation's toolkit that can be used to improve the way human groups engage with efforts to conserve threatened biodiversity. Here we review the various forms of anthropomorphism and their uses, as well as the processes by which animals are anthropomorphized. Understanding the formation and uses of anthropomorphism provides a guide to applying the benefits of anthropomorphism to the conservation of any species. We also contrast this to the non-anthropomorphic, non-anthropocentric views of other species current in non-Western cultures. Finally, we discuss the potential negative outcomes of anthropomorphism in conservation, and suggest how these could be managed.

\section{Defining anthropomorphism}

In order to understand the roles of anthropomorphism in conservation, we need to acknowledge the lack of a consistently understood definition of the term. Most dictionaries broadly define anthropomorphism as the attribution of human characteristics to nonhuman 
entities. Traditionally, anthropomorphism has been used to refer to the overestimation/ misattribution/inappropriate/inaccurate attribution of uniquely/properly human traits (Guthrie 1997). Because the notion of "human" is central to the concept of anthropomorphism, it would stand that in order to fully understand what anthropomorphism means, one must first understand what it means to be human as separate from all other entities (Emel 1995). Scholars have debated what it means to be uniquely human for millennia. Proposed points of delineation between human and nonhuman have included issues of morphology, language, symbolic communication, rational autonomy, sentience, and consciousness (among others). As we continue to discover new truths about nonhuman animals, scholars continue the debate and search for a uniquely human characteristic. Even the one similarity among anthropomorphism definitions-a comparison to humans-is a poorly understood concept. Without this understanding, it would seem unjustified to make judgments on the appropriateness of the attribution of human characteristics, as has been suggested in traditional definitions. Furthermore, without a universally-held understanding of a human characteristic, the operationalization of anthropomorphism is subjected to individual interpretations of what constitutes a human attribution or characteristic (cf. Taylor 2011; Milton 2005). Thus, the debate over the acceptability of anthropomorphizing an animal is confused by various conceptualizations of that action.

Anthropomorphizing can take many forms. These vary on a continuum from weak forms, such as identifying similarities between ourselves and the anthropomorphized object (Guthrie 1997) or speaking metaphorically of a nonhuman object, to stronger forms of anthropomorphism whereby the person behaves and endorses the personally-held belief that the non-human agent has humanlike characteristics or traits (Epley et al. 2007). Scholars use a variety of indicators for when anthropomorphism is occurring. Representations of animals could become more human-like in a physical sense, making attributions of human physical features like forward-facing eyes or walking upright (e.g. Nowak and Rauh 2008). Attributions of human cognition and emotions are also types of anthropomorphisms seen in the literature (e.g. Serpell 2003; Ikeda et al. 2004). Anthropomorphisms can also take the form of animals taking on human cultural characteristics, such as animals wearing clothes or engaging in a tea party (e.g., Allen et al. 1994; Antonacopoulos and Pychyl 2008).

Any one of these anthropomorphism indicators can also vary in intensity. For example, a drawing of a horse with eyes facing forward (instead of on the side) is a smaller type of physical anthropomorphism than a horse with eyes facing forward and standing on two feet. The up-right horse could be further anthropomorphized by adding another type of anthropomorphism, such as the horse dressed in clothes or playing golf. The anthropomorphisms depicted in a drawing are limited in comparison to the possibilities for full character development in an anthropomorphized feature film (e.g., Finding Nemo). The diversity of individually-held conceptualizations of "human" and representations of humanlike characteristics suggest that anthropomorphism can be operationalized in many ways.

Not all forms of anthropomorphism develop in the same way or under the same conditions, nor do they all have the same social roles or practical uses (Fig. 1). For example, a hunter may attribute strategic thinking and emotions to their prey as a way of understanding and solving the problem of killing it (Kennedy 1992; Mithen 1996; Manfredo and Fulton 2008). Representations of animals wearing clothes and engaging in cultural activities have historically been a way to obliquely discuss politics and social life (e.g., Oerlemans 2007). Choosing between the potential functions of anthropomorphization is one task for conservationists who wish to use it as a tool. 


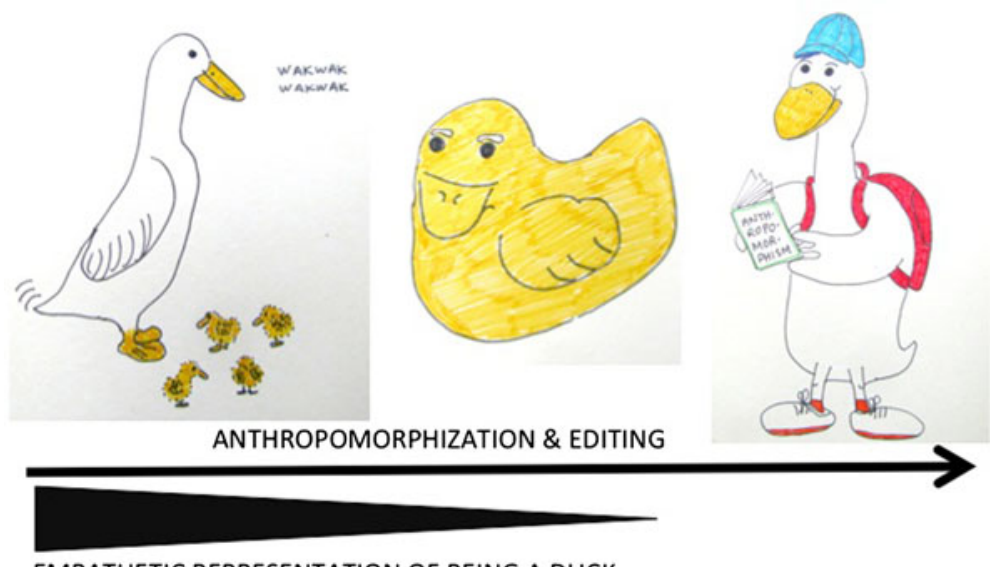

EMPATHETIC REPRESENTATION OF BEING A DUCK

\section{ABSTRACTED FEATURES RELEVANT TO SOCIAL USES}

HUMAN PHYSICAL FEATURES, CULTURAL ITEMS AND SOCIAL NARRATIVES

Fig. 1 A schematic showing the interactions between different elements of anthropomorphization and the associated editing of nonhuman species representations. Far left, a domestic mother duck cares for her recently hatched ducklings by interacting with them through movements and sounds. This representation supports communication of the experience of being a duck, and teaching waterfowl natural history. Middle, a rubber duck toy has some key elements of real ducks (e.g. yellow color of ducklings, wings, bill, floating behavior), but it is missing others (e.g. legs, most other behaviors) and has some non-duck, human-like features (e.g. eyebrows, forward facing eyes). This combination of features supports playing with the rubber duck in a bath. Through play, children may add additional elements of empathetic anthropomorphism. Far right, Daphne the Duck is taking a class on anthropomorphism at summer school. She has some key elements of duck anatomy as well as several human-specific anatomical features, human cultural items and practices, and an implicit social narrative (going to school). This set of features enables Daphne to communicate the importance of studying anthropomorphism. Famous highly anthropomorphised ducks include Donald Duck and Beatrix Potter's Jemima Puddle-Duck. Daphne the Duck would normally be considered the most anthropomorphized duck here, but the mother duck can also be seen as highly anthropomorphized in an egomorphic sense. Images copyright M.R.-B. (Color figure online)

\section{How animals are anthropomorphized}

Anthropomorphism can develop from several different types of perceived similarity with species. Empathy is commonly referred to as an outcome of anthropomorphism (e.g. Chan 2012) but can also be thought of as a basis for anthropomorphizing a species. Many authors define empathy broadly as a process of intuitively understanding the logic behind the known behaviors of another species or nonhuman entity (Root-Bernstein and Root-Bernstein 1999). This kind of empathy can be the origin of our understanding of the non-human species, which can then be compared to humans and used to recognize or speculate about anthropomorphic features.

Lorimer (2007) has described a set of engagements with non-human animals that produce non-human charismas. Charismatic species have characteristics that gain sensual and emotional salience for humans due to the type of interaction or experience that the human has with the non-human. Among other types of charisma, Lorimer (2007) defines an 
anthropomorphic charisma based on a recognition of features shared with humans, such as care of young, pair bonding or playing. Yet all forms of non-human charisma allow us to make comparisons to humans, and thus anthropomorphize. For example, people engage with bitterns primarily through the sound of their calls in their habitat (an "ecological charisma"). The loud "boom" of the otherwise cryptic bittern forms the basis for anthropomorphized representations emphasizing bitterns' strength and similarity to a marching band (Barua and Jepson 2010).

Finally, egomorphism is an important engagement with non-human species that is closely related to anthropomorphism. Egomorphism is defined as the perception that another species has self-like, rather than human-like, qualities (Milton 2005). If anthropomorphism suggests that other species become persons through metaphor, egomorphism posits that they already share fundamental aspects of person- or selfhood with ourselves. One could egomorphize a spider by considering it to be a sentient being with a life history and a personal memory. Thus, egomorphism, like empathy and non-human charisma, are forms of engagement that construct an understanding of what it is to be, become, or sense another species. Anthropomorphization acts on these engagements.

People construct anthropomorphic meanings around other species in many ways. These may include personal interactions with individuals of a non-human species, interactions with representations of species created by institutions such as flagship species or logos (Barua pers. comm.), cultural interactions in which representations of a species play a symbolic role or provide a function (e.g. a toy to play with), or in which a species plays a role as a legitimate focus of some social activity (e.g. telling an anecdote), and variations on these. For example, pet owners develop representations of what those pets like, want, understand, and have tendencies to do. This may have several anthropomorphic outcomes, such as empathy for the pet's feelings, the use of agentive language to describe the pet's behavior, and the inclusion of the pet as an actor in certain social interactions (e.g. Serpell 2003). Hunters, herders, birders, naturalists, field biologists and other stakeholders in natural habitats may also anthropomorphize. These people spend long periods of time experiencing the same conditions as the species they are guiding or seeking. In this way, they develop an empathetic understanding of how other species behave and react-fearfully, gracefully, playfully and so on-through sharing of experiences (Ingold 2000; Sapolsky 2001; Lorimer 2006; Candea 2010).

Many people develop anthropomorphic understandings of species through their representations rather than through interactions in nature. Cultural products that include, for example, representations of pandas, range from the World Wildlife Fund (WWF) logo to nature documentaries, from cheese commercials (i.e. Panda Cheese) to plush toys. Each of these represents only some of all possible attributes of real pandas, and may add humanlike attributes. These edited and anthropomorphized pandas are either deliberately designed or culturally evolved to suit social, cultural and economic roles and desires (Brown 2010). One example is the WWF logo, where the panda was modified over time to mirror the change in the NGO's structure, from what was initially a shoe-string outfit to a professionalized organization with an increasingly corporate structure (Nicholls 2011). Another example is the way the sexual and reproductive behaviors of the two pandas at the National Zoo in Washington D.C. were covered by the press, using language used to describe human sexuality, allegorizing panda behaviors in terms of contemporary human social issues and mores in attempts to dramatize the story to promote public identification with the pandas. However, the human cultural representations of the mating process do not adequately describe natural panda mating behaviors. While the language used in the press represented the pandas' mating behaviors in a way that was easily identifiable to humans, it did not 
promote an understanding of the species true to its natural behavior (Chris 2006). Hypothetically, a greeting card company might consequently see pandas as an efficient and affecting conveyor of a "congratulations on your new baby" message, and might legitimize, contextualize or increase the effectiveness of the panda in this social role by depicting two panda parents holding hands, leaning over a baby panda in a stroller. This process of editing away non-human features and adding humanlike features can be thought of as an "anthropomorphic creep."

Marketing can be used to deliberately design and position a representation of a nonhuman species for particular ends. Conservationists already use flagship species to promote conservation actions (e.g. Krauss 2005; Smith and Sutton 2008; Veríssimo et al. 2009; Barua et al. 2010; Barua et al. 2011; Veríssimo et al. 2011; Root-Bernstein and Armesto 2013), and though anthropomorphic traits such as forward facing eyes are often key in flagship selection (Smith et al. 2012), little attention has been given to the role of anthropomorphized flagships. Commercial marketers have long established that anthropomorphism can be an effective way to connect people to products and services. This has led to the use of anthropomorphism in campaigns dealing with products ranging from flavored fruit drinks to condoms to car parts (Spears et al. 1996; Waytz et al. 2010). Nonetheless, marketers have realized anthropomorphism is not universal, with its impact influenced by the social, economic and cultural profile of the target audience. As such, anthropomorphism has been used largely in a strategic way for particular product and service categories and linked to specific animal groups (Epley et al. 2008; Waytz et al. 2010). For example, representations of animals are mainly associated with the selling of food and drink (nondurables), pet foods, and services, with wild animals more frequently shown in an anthropomorphic state than domesticated animals (Spears et al. 1996). Social marketers have also used anthropomorphism to improve the impact of conservation messages. For example, in the United States, Smokey the Bear, a black bear shown in a Forest Ranger's uniform, is one of the most popular conservation icons, branded with his message "only you can prevent wildfires." As would be expected, anthropomorphism is common in marketing campaigns that associate animals to the brands they are promoting as a means to influence their target audience (Spears et al. 1996). This influence occurs both through the symbolic meanings that have been culturally assigned to particular animal species as well as the species physical attractiveness and likability (Lancendorfer et al. 2008). In this context, anthropomorphism gives marketers ample flexibility to move away from or reinforce the symbolic meanings associated with a species and in this way construct brand personalities that more effectively resonate with their target audience (Kotler and Armstrong 2012). Nevertheless, we still do not understand many of the dimensions of this use such as what aspects of animals (e.g. behavior, physical) are most often anthropomorphized and how these different aspects impact different socio-economic groups.

Anthropomorphism is thus likely to motivate conservation support by highlighting commonalities between the human and non-human conditions. Anthropomorphism is based on at least three primary engagements with other species, including egomorphism, charisma and empathy, but it can develop through different experiences and take many forms.

\section{Anthropormorphism and non-Western cultures}

The previous section largely considered anthropomorphization from a Western cultural vantage point. Anthropomorphic representations presuppose that people think of humans as 
forming a referential and distinct category from non-humans. After all, we are not writing this article about how to position species we wish to conserve as panda-morphic, or sea turtle-morphic, or tree-morphic, despite the considerable conservation traction that these taxa may possess. Anthropomorphic representations are transgressive and/or transformative, and thus powerful, in the context of Western anthropocentrism and the nature/culture and human/animal dualisms (Ingold 1994; Descola 1996; Fréger 2012). Within this cultural framework, distrust of anthropomorphism as a mode of scientific thinking drew on the idea that non-humans had no mental or emotional states, or that these could not be known (Burkhardt 2005). Anthropomorphism was thus represented as fantasy all across its spectrum (see Fig. 1), firmly on the culture side of the nature/culture dualism.

Non-Western cultures, by contrast, display a "seemingly infinite empirical diversity of nature-culture complexes" (Descola 1996 p. 84). Descola divides these complexes into three main types, naturalism (e.g. Western thought), animism (e.g. non-humans speaking to humans), and totemism (e.g. kinship between humans and non-humans). In totemic and animistic complexes, anthropomorphism per se is a non-concept. For example, identification of orangutans as human-like persons by Western visitors to orangutan conservation centers in Malaysia can result in a strong emotional bond that rewards conservationoriented caring through volunteerism (Parreñas 2012). This empathetic egomorphization constructs a hybrid orangutan/human actor that "disrupts" nature vs. culture while also linking these categories through the "fluid nature of identification" with the orangutan (Sowards 2006; see Descola 1996). The emotional bond is arguably motivating and rewarding in part because it both creates and resolves the problem of orantugan-human similarity. By contrast, indigenous Indonesians already know that orangutans are kin. In their totemic conception, orangutans are humans who went to live in the forest, and they remain human (Sowards 2006). Anthropomorphization of orangutans for conservation outreach to this indigenous community might not produce a similar emotional bond of caring: what would it mean to anthropomorphize a person? The process of anthropomorphization of orangutans could have significantly different meanings across cultures.

Many indigenous cultures have some form of totemic or animistic conception of what humans are. For example, in tropical South America monkeys are often a kind of human, or descendants of humans (Cormier 2006). Throughout the Americas, indigenous peoples have been characterized as understanding humans to be what animals and spirits know themselves as when they are at home (de Castro 1998). Their animal attributes are like coats that they take on and off (de Castro 1998). Such conceptions do not necessarily lead to any particular set of taboos, hunting practices, or ritual interactions, which can vary widely despite similar beliefs (Descola 1996). It is important to recognize that within animistic or totemic complexes, representations of other species talking to or marrying humans are not imaginary constructions inhabiting fantasy worlds, as anthropomorphized animals may be in Western thought. Although metaphor and ritual may be important for making spaces in which non-humans can communicate or act as kin, within these spaces humans experience a reality of other species (Descola 1996; Ingold 2000; Rival 2012). Experientially, this may be similar to a Western person's conviction that people with whom we only ever speak via telephone really exist. Since in these cultures some nonhuman taxa are considered people, anthropomorphizing them is not necessary. At the same time, anthropomorphization of species not considered people may also not make sense in the cultural context, since kinship or ritual communication are the ways in which other taxa are understood to be persons.

Forms of reciprocity are also a common way in which humans interact with non-human taxa, via for example revenge on human hunters (human predation) or trans-generational 
position swapping (e.g. reincarnation) (de Castro 1998). Hunting and gathering is thus not simply a 'traditional practice' but also a way of being a human in the world, and perhaps an obligation. Thus in situations where conservationists wish to reduce or eliminate take of a species, indigenous communities may not be able to conceptualize this withdrawal from interaction as an act of caring (Collomb 2009; Roué 2009). This is because in caring about other species they see them as having person-like qualities or social roles-social roles in which one kind of person eats another. Anthropormorphization of non-human species in the West for conservation purposes tends to imply, by contrast, that because other species are human-like, they deserve personal autonomy, personal space, and freedom from suffering and death, all of which humans are seen to impede.

If one goal of anthropomorphizing species for conservation purposes is to reduce anthropocentrism in the engagements with biodiversity by members of Western or Westernized cultures, one might ask whether anthropomorphism could approximate an animistic or totemic complex. This seems unlikely: anthropomorphism can bridge the dualisms of Western thought for particular ends, but is not a substitute for a completely elaborated worldview. Further, non-anthropocentric, non-dualist ways of thinking do not necessarily promote conservation-friendly actions. On the one hand, this is because how people behave towards other people (of whatever kind) is a complex issue. On the other hand, our notions of environmental stewardship and compassion towards other species are themselves situated within a dualist worldview. In the next section we consider other limitations of anthropomorphism as a tool for conservation.

\section{Potential negative outcomes of anthropomorphism as a conservation tool}

Here we discuss three kinds of negative outcomes of anthropomorphizing non-human species. In the first kind, an apparently positive outcome conflicts with conservation goals. In the second kind, animals violate the social expectations raised by their anthropomorphization, creating conflict with humans. Finally, non-human species can take on pejorative social stereotypes, with negative effects on their conservation.

A main goal of using empathetic anthropomorphism as a conservation tool is to promote care and protection of individuals of a species. But producing a caring attitude towards individual non-humans can negatively affect conservation goals. Research to promote humans caring for other humans shows that willingness to contribute to humans in need is greatest when the information given with the request for help is focused on a single individual identified with a picture (Kogut and Ritov 2005). Slovic (2007) claims that most people will exert great effort to help alleviate individual suffering. These same people, however, can become "numbly indifferent to the plight of individuals who are "one of many' in a much greater problem" (p. 79). Slovic (2007) provides cattle and canine examples of how this phenomenon also functions with human perceptions of nonhuman animals. The feeling of indifference and associated lack of action begins at two individuals (Slovic 2007). Because anthropomorphism can draw people's attention to individuals, it is equipped to heighten care. Further research is needed, however, to determine whether anthropomorphism is effective or destructive in teaching caring actions for complex concepts, such as ecosystems and biodiversity.

As Chan (2012) notes, a caring attitude directed at individuals rather than systems can act as a limitation to conservation. Chan (2012) cites a hypothetical example whereby anthropomorphizing one species heightens care for that species and leads to public support for the killing of a competitor or predator species. Another possibility is that a caring 
attitude would conflict with conservation actions such as control of zoo populations in breeding programs, culling, trapping or tagging. As a case in point, breeding programs for threatened species in zoos are divided about whether it is better to prevent unwanted crosses entering the gene pool through the use of contraceptives (more efficient), or by allowing animals with unplanned pregnancies to experience natural offspring-raising behaviors, followed by euthanizing these offspring when they reach adulthood (argued to be more caring) (Kaufman 2012). Development of a caring attitude might also conflict with the promotion of conservation as an investment in the intrinsic or biodiversity values of species-investments that are ethically or pragmatically advisable whether one has empathy for the species in question or not. For example, if marketing of anthropomorphized representations increases caring towards species A, this might be at the expense of conservation actions in support of the ecologically important, but unmarketed and thus uncared for, species B (see e.g. Smith et al. 2012). In addition, caring for an individual or species can compromise overall species and/or habitat conservation objectives. Take for example the behavioral outcomes following the release of the animated film Finding Nemo. Using anthropomorphism, viewers grew to care for the marine characters, especially Nemo, a juvenile clownfish (from the genus Amphiprion). After the movie's release, there was a reported increase in the demand for clownfish in the aquarium trade industry (Harley 2005). This has led to overfishing on the reefs (Yong et al. 2011). In this case, the caregiving behavioral outcome has led to a negative conservation outcome.

Anthropomorphism can also backfire by setting up expectations of human-like social behavior that non-human species cannot satisfy. For example, Japanese tourists at monkey feeding parks understand the feeding interaction as akin to Japanese gift-giving traditions (Knight 2005). However, the tourists are often upset that monkeys also steal food and fight with one another to access it, which they understand as a rude violation of the meaning of the feeding interaction. In another example, northern Portuguese farmers address curses to wild boar that raid their fields (Galhano-Alves 2004). Engaging wild boar in a social practice (ritual, audible cursing) suggests that the wild boar are considered to be persons violating a social pact (cf. Theodossopoulos 2005). Finally, in Japan non-native raccoons (Procyon lotor) are now a serious source of human-wildlife conflict in both residential and agricultural lands, as well as historical and biologically important sites. Hundreds of raccoons were imported into Japan following a smash hit animated cartoon series Rascal Raccoon during the late 1970s to early 1980s. The popular cartoon series anthropomorphized the North American raccoon as harmless, cute and humorous, and a faithful human companion with enviable hygiene and that cared for children. Japanese households with raccoons, however, experiencing the natural behavior of Procyon lotor eventually released their pet raccoons into the wild, precipitating the need for a costly ongoing nation-wide intensive raccoon eradication program (Ikeda et al. 2004). Holding other species to social norms that they cannot fulfill can create conservation problems or could hinder support for conservation actions on their behalf.

Finally, being human-like is not necessarily a good thing, and non-human species sometimes acquire negative social stereotypes. For example in Chile a naturalized archaeophyte tree called the espino (Acacia caven) can be anthropomorphized as stoic and plebian (Root-Bernstein 2012). The espino's close association with human poverty also leads to it being described as an agent of degradation that has "parasitized" the landscape and should be replaced with productive non-native trees, an attitude that mirrors the ambivalence towards local peasants (Aronson et al. 1998). Sport fishermen in the United States view otters as their direct competitors, and characterize them as gluttonous individuals who kill for fun, something seen as unnatural and requiring regulations comparable 
to those to which the human fishers must adhere (Goedeke 2005). Similarly wolves in North America have acquired significant social stereotypes as murderous blood-thirsty vampires (Emel 1995). These stereotypes have been successfully reinforced through film and popular culture. On the Caribbean island Dominica, the power of social marketing and anthropomorphizing a species is further illustrated by findings that show that fetishising anthropomorphized species used as conservation flagships may marginalize other closely related species within local culture. In this case, the publics' emotional investment developed in the Imperial Parrot (Amazona imperialis), the national bird and conservation flagship of the nation, led to the sister species, the Red-necked Parrot (Amazona arausiaca) being perceived as the flagship's undeserving competitor. Here, the anthropomorphized flagship became increasingly associated with positive cultural stereotypes such as beauty and sophistication, while the sister non-flagship species was denigrated as unappealing and less worthy of conservation investment. Most importantly, these anthropomorphized constructions reflected stereotypes of gendered, racial and classist identities of Dominican culture, which significantly influenced the conservation behavior of local residents, including law enforcement officers (Douglas 2011).

In summary, anthropomorphization can encourage undesirable behaviors or expectations about the character of interactions between humans and non-humans. The lesson here is that when planning how to anthropomorphize a species, remember that being human-like means being a member of a society. People may expect the non-human to engage in human social relations, or they may metaphorically see their society reflected in the species' ecosystem. A proactive way for conservationists to deal with potential problems would be to anthropomorphize the target species in contexts that illustrate model interactions with both humans and key non-human species with which the target species may be associated.

\section{Conclusions}

Any species may be anthropomorphized, in various ways, within the Western dualistic tradition. Some authors have urged caution, taking the position that a broad application of anthropomorphization for conservation ends would be "irresponsible" (Chan 2012). By contrast, we believe that it would be irresponsible to limit the use of this tool to a small percentage of species and a handful of traits selected without reference to social science. A problem with tying "legitimate" anthropomorphism to a "hard" basis in biological experiments demonstrating "bona fide" human-likeness is the strong anti-anthropomorphic, human-exceptionalism bias in biological sciences (Mitchell 1997). Such experiments use conceptual and methodological criteria of similarity to humans unrelated to the ways in which people judge anthropomorphism in everyday life. Anthropomorphizing species as egomorphic objects of empathetic insight is a typical outcome of personal interactions with non-humans, and is often associated with being a stakeholder in natural habitats, at least in Western cultures. Guiding and promoting such anthropomorphizations as tools for conservation is likely to be efficient and effective. We acknowledge that when dealing with cultural representations of non-human species, anthropomorphic creep could be problematic. But under what conditions? The depiction of racoons that led Japanese households to adopt them as family pets which were later introduced into the wild was an example of anthropomorphic creep with unintended consequences. But is Smokey the Bear less effective as a representative of the danger posed to the forest ecosystem by fires because he is wearing a forest ranger's uniform? Does Smokey the Bear's uniform undermine bear conservation messages? This is not clear. 
We suggest that an appropriate way to anthropomorphize a species for conservation purposes is to (1) emphasize the characteristics the species already possesses that people engage with during personal interactions that form the egomorphic, empathetic and charismatic bases for anthropomorphization, and (2) give the species just enough recognizably human-like characteristics to make it a credible and positive social actor, given its intended role. Extrapolating from Spears et al.'s (1996) observations on the marketing uses of domestic and wild animals, species that often interact with the target audience can be strongly egomorphized, while species that the audience has limited personal experience with may particularly benefit from the addition of some human-like features. Establishing best practice for implementing these recommendations, while avoiding potential negative outcomes of anthropomorphization, requires further research, especially in social sciences and marketing.

Acknowledgments MR-B is funded by a Post Doctoral Research Fellowship from FONDECYT (No. 3130336). LD is funded by the Center for Biodiversity and Conservation of the American Museum of Natural History (AMNH) in affiliation with Columbia University. DV is funded by the Doctoral Programme (SFRH/BD/60993/2009) of the Fundação para a Ciência e Tecnologia.

\section{References}

Allen JS, Park J, Watt SL (1994) The chimpanzee tea party: anthropomorphism, orientalism, and colonialism. Vis Anthropol Rev 10(2):45-54

Antonacopoulos NMD, Pychyl TA (2008) An examination of the relations between social support, anthropomorphism and stress among dog owners. Anthrozoos 21(2):139-152

Aronson J, del Pozo A, Ovalle C, Avendaño J, Lavin A, Etienne M (1998) Land use changes and conflicts in central Chile. In: Rundel PW, Jaksic FM (eds) Landscape disturbance and biodiversity in Mediterranean-type ecosystems. Springer, New York

Barua M, Jepson P (2010) The bull of the bog: Bittern conservation practice in a Western bio-cultural setting. In: Tidemann S, Gosler A (eds) Ethno-ornithology: birds, indigenous peoples, culture and society. Earthscan, London, pp 301-312

Barua M, Tamuly J, Ahmed RA (2010) Mutiny or clear sailing? Examining the role of the Asian Elephant as a flagship species. Hum Dimens Wildl 15:145-160

Barua M, Root-Bernstein M, Ladle R, Jepson P (2011) Defining flagship uses is critical for flagship selection: a critique of the IUCN climate change flagship fleet. Ambio 40(4):431-434

Brown S (2010) Where the wild brands are: some thoughts on anthropomorphic marketing. Mark Rev 10(3):209-224

Burkhardt RW Jr (2005) Patterns of behavior: Konrad Lorenz, Niko Tinbergen and the founding of ethology. University of Chicago Press, Chicago

Candea M (2010) "I fell in love with Carlos the meerkat": engagement and detachment in human-animal relations. Am Ethnol 37(2):241-258

Chan AAY-H (2012) Anthropomorphism as a conservation tool. Biodivers Conserv 21:1889-1892

Chris C (2006) Watching wildlife. University of Minnesota Press, Minneapolis

Collomb G (2009) "Sous les tortues, la plage?" Protection de la nature et production des territoires en Guyane. Ethnol Française 39(1):11-21

Cormier L (2006) A preliminary review of neotropical primates in the subsistence and symbolism of indigenous lowland South American peoples. Ecol Environ Anthropol 2(1):14-32.

de Castro EV (1998) Cosmological Deixis and Amerindian Perspectivism. J R Anthro Inst 4:469-488

Descola P (1996) Constructing natures: symbolic ecology and social practice. In: Descola P, Pálsson G (eds) Nature and society: anthropological perspectives. Routledge, London, pp 82-102

Douglas L (2011) The social and ecological underpinnings of human-wildlife conflict on the island of Dominica. Dissertation, Columbia University

Emel J (1995) Are you man enough, big and bad enough? Ecofeminism and wolf eradication in the USA. Environ Plan D-Soc Space 13:707-734

Epley N, Waytz A, Cacioppo JT (2007) On seeing human: a three-factor theory of anthropomorphism. Psychol Rev 114(4):864-886 
Epley N, Waytz A, Akalis S, Cacioppo JT (2008) When we need a human: motivational determinants of anthropomorphism. Soc Cogn 26:143-155

Fréger C (2012) Wilder Mann ou la figure du sauvage. Thames \& Hudson, Paris

Galhano-Alves JP (2004) Man and wild boar: a study in Montesinho Natural Park, Portugal. Galemys 16(special):223-230.

Goedeke TL (2005) Devils, angels or animals: the social construction of otters in conflict over management. In: Herda-Rapp A, Goedeke TL (eds) Mad about wildlife: looking at social conflict over wildlife. Brill, Boston, pp 25-50

Guthrie SE (1997) Anthropomorphism: a definition and theory. In: Mitchell RW, Thompson NS, Miles HL (eds) Anthropomorphism, anecdotes, and animals. State University of New York Press, Albany, pp 50-58

Harley, W (Producer) (2005, 10 January) Vanuatu-Saving Nemo [online documentary]. ABC Australia: Journeyman Pictures. Accessed online: http://www.journeyman.tv/18050/short-films/saving-nemo.html and http://www.youtube.com/watch?v=rC8rkMjIZAk

Ikeda T, Asasno M, Matoba Y, Abe G (2004) Present status of invasive alien raccoon and its impact in Japan. Glob Environ Res 8:125-131

Ingold T (1994) Introduction. In: Ingold T (ed) What is an animal? Routledge, London, pp 1-16

Ingold T (2000) The perception of the environment. Essays on living, dwelling and skill. Routledge, London

Kaufman L (2012) When babies don't fit plan, question for zoos is, now what? The New York Times, Science Section August 2. Accessed online: http://www.nytimes.com/2012/08/03/science/zoos-divideover-contraception-and-euthanasia-for-animals.html?hp.

Kennedy JS (1992) The new anthropomorphism. Cambridge University Press, Cambridge

Knight J (2005) Feeding Mr. Monkey: cross-species food "exchange" in Japanese monkey parks. In: Knight $\mathrm{J}$ (ed) Animals in person: cultural perspectives on human-animal intimacies. BERG, Oxford, pp 231-253

Kogut T, Ritov I (2005) The "identified victim" effect: an identified group, or just a single individual? J Behav Decis Making 18:157-165

Kotler P, Armstrong G (2012) Principles of marketing. Pearson Prentice Hall, Upper Saddle River

Krauss W (2005) Of otters and humans: an approach to the politics of nature in terms of rhetoric. Cons Soc 3(2):354-370

Lancendorfer KM, Atkin JL, Reece BB (2008) Animals in advertising: love dogs? Love the ad! J Bus Res 61:384-391

Lorimer H (2006) Herding memories of humans and animals. Environ Plan D: Soc Space 24:497-518

Lorimer J (2007) Nonhuman charisma. Environ Plan D: Soc Space 25:911-932

Manfredo MJ, Fulton DC (2008) The biological context of wildlife values: are there etchings on the slate? In: Manfredo MJ (ed) Who cares about wildlife?. Springer, New York, pp 29-48

Milton K (2005) Anthropomorphism or egomorphism? The perception of nonhuman persons by human ones. In: Knight $\mathrm{J}$ (ed) Animals in person: cultural perspectives on human-animal intimacies. BERG, Oxford, pp 255-271

Mitchell RW (1997) Anthropomorphic anecdotalism as method. In: Mitchell RW et al (eds) Anthropomorphism, anecdotes, and animals. SUNY Press, Albany, pp 151-169

Mithen S (1996) The prehistory of the mind. Thames and Hudson Ltd., London

Nicholls H (2011) The art of conservation. Nature 472:287-289

Nowak KL, Rauh C (2008) Choose your "buddy icon" carefully: the influence of avatar androgyny, anthropomorphism and credibility in online interactions. Comput Hum Behav 24(4):1473-1493

Oerlemans O (2007) A defense of anthropomorphism: comparing Coetzee and Gowdy. Mosaic 40(1): $128-196$

Parreñas RJS (2012) Producing affect: transnational volunteerism in a Malaysian orangutan rehabilitation center. Am Ethnol 39(4):673-687

Rival L (2012) Animism and the meanings of life: reflections from Amazonia. In: Brightman M, Grotti VE, Ulturgasheva $\mathrm{O}$ (eds) Animism in rainforest and tundra: personhood, animals, plants and things in contemporary Amazonia and Siberia. Berghahn Books, United States, pp 69-81

Root-Bernstein M (2012) Ecosystem engineering in the degu Octodon degus with applications to conservation. PhD Thesis, Pontificia Universidad Católica de Chile, Santiago

Root-Bernstein M, Armesto J (2013) Selection and implementation of a flagship fleet in an undervalued region of high endemicity. Ambio, early view

Root-Bernstein RS, Root-Bernstein MM (1999) Sparks of genius. Houghton Mifflin, Boston

Roué M (2009) “Une oie qui traverse les frontières”La bernache du Canada. Ethnol Française 39(1):23-34

Sapolsky RM (2001) A primate's memoir: a neuroscientist's unconventional life among the baboons. Simon and Schuster, New York 
Serpell JA (2003) Anthropomorphism and anthropomorphic selection-beyond the 'cute response'. Soc Anim 11(1):83-100

Slovic P (2007) "If I look at the mass I will never act": psychic numbing and genocide. Judg Decis Making 2(2):79-95

Smith AM, Sutton SG (2008) The role of a flagship species in the formation of conservation intentions. Human Dimens Wildlife 13(2): 127-140

Smith RJ, Veríssimo D, Isaac NJB, Jones KE (2012) Identifying Cinderella species: uncovering mammals with conservation flagship appeal. Cons Lett 5:205-212

Sowards SK (2006) Indentification through orangutans: destabilizing the nature/culture dualism. Ethics Environ 11(2): 1085-6633

Spears NE, Mowen JC, Chakraborty C (1996) Symbolic role of animals in print advertising: Content analysis and conceptual development. J Bus Res 37:87-95

Tam K-P, Lee S-L, Chao MM (2013) Saving Mr. Nature: anthropomorphism enhances connectedness to and protectiveness toward nature. J Exp Soc Psychol 49:514-521

Taylor N (2011) Anthropomorphism and the animal subject. In: Boddice R (ed) Anthropocentricism: humans, animals, environments. Brill, Leiden, pp 265-279

Theodossopoulos D (2005) Care, order and usefulness: the context of a human-animal relationship in a Greek island community. In: Knight $\mathbf{J}$ (ed) Animals in person: cultural perspectives on human-animal intimacies. BERG, Oxford, pp 15-35

Veríssimo D, Fraser I, Groombridge J, Bristol R, MacMillan DC (2009) Birds as tourism flagship species: a case study of tropical islands. Anim Cons 12(6):549-558

Veríssimo D, MacMillan DC, Smith RJ (2011) Towards a systematic approach for identifying conservation flagships. Cons Lett 4(1):1-8

Waytz A, Cacioppo J, Epley N (2010) Who sees human? Persp Psychol Sci 5:219-232

Yong DL, Fam SD, Lum S (2011) Reel conservation: can big screen animations save tropical biodiversity? Tropic Cons Sci 4(3):244-253 